\title{
Yang-Mills as Massive Chern-Simons Theory: A Third Way to Three-Dimensional Gauge Theories
}

\author{
Alex S. Arvanitakis, ${ }^{1, *}$ Alexander Sevrin, ${ }^{2, \dagger}$ and Paul K. Townsend ${ }^{1, \$}$ \\ ${ }^{1}$ Department of Applied Mathematics and Theoretical Physics, Centre for Mathematical Sciences, \\ University of Cambridge, Wilberforce Road, Cambridge CB3 OWA, United Kingdom \\ ${ }^{2}$ Theoretische Natuurkunde, Vrije Universiteit Brussel, and The International Solvay Institutes, \\ Pleinlaan 2, B-1050 Brussels, Belgium and Physics Department, Universiteit Antwerpen, \\ Campus Groenenborger, 2020 Antwerpen, Belgium \\ (Received 4 February 2015; published 7 May 2015)
}

\begin{abstract}
The Yang-Mills (YM) equation in three spacetime dimensions (3D) can be modified to include a novel parity-preserving interaction term, with an inverse mass parameter, in addition to a possible topological mass term. The novelty is that the modified YM equation is not the Euler-Lagrange equation of any gauge-invariant local action for the YM gauge potential alone. Instead, consistency is achieved in the "third way" exploited by 3D minimal massive gravity. We relate our results to the "novel Higgs mechanism" for Chern-Simons gauge theories.
\end{abstract}

In three spacetime dimensions (3D) the general gaugeinvariant second-order action for a Yang-Mills (YM) gauge potential $A$ is

$$
I_{\mathrm{TMYM}}[A]=\frac{1}{2 g^{2}} \int d^{3} x \tilde{F}^{\mu} \cdot \tilde{F}_{\mu}+\frac{\mu}{g^{2}} I_{\mathrm{CS}}[A],
$$

where $\tilde{F}$ is the dual Yang-Mills field strength,

$$
\tilde{F}^{\mu}=\varepsilon^{\mu \nu \rho}\left(\partial_{\nu} A_{\rho}+\frac{1}{2} A_{\nu} \times A_{\rho}\right),
$$

and $I_{\mathrm{CS}}[A]$ is the Chern-Simons action

$$
\frac{1}{2} \int d^{3} x \varepsilon^{\mu \nu \rho}\left[A_{\mu} \cdot \partial_{\nu} A_{\rho}+\frac{1}{3} A_{\mu} \cdot A_{\nu} \times A_{\rho}\right] .
$$

Here we suppose, for simplicity, that the gauge group is $S U(2)$ and we use vector algebra notation for products of $S U$ (2) triplets; the generalization to other gauge groups is straightforward.

For $\mu=0$, the action (1) is the 3D YM action with coupling constant $g$. Notice that $g^{2}$ has dimensions of mass, so $\mu / g^{2}$ is dimensionless. For nonzero $\mu$ the action is that of topologically massive Yang-Mills (TMYM) theory [1,2], which propagates an $S U(2)$ triplet of spin-1 modes of mass $\mu$. The field equation is

$$
\varepsilon^{\mu \nu \rho} D_{\nu} \tilde{F}_{\rho}+\mu \tilde{F}^{\mu}=0,
$$

where $D_{\mu}$ is the covariant derivative, defined such that

$$
D_{\mu} V=\partial_{\mu} V+A_{\mu} \times V
$$

for any $S U(2)$-triplet $V$.
Let us now add a source current $J$ to the right-hand side of Eq. (4), so that

$$
\varepsilon^{\mu \nu \rho} D_{\nu} \tilde{F}_{\rho}+\mu \tilde{F}^{\mu}=J^{\mu} .
$$

Because of the Bianchi identity $D_{\mu} \tilde{F}^{\mu} \equiv 0$, consistency requires the source current to be covariantly conserved:

$$
D_{\mu} J^{\mu}=0
$$

There are two standard ways to construct a source current with this property: $1 . J=j(\phi)$, the Noether current in a YM background for lower-spin fields $\phi$. In this case, $D_{\mu} j^{\mu}(\phi)=0$ as a consequence of the $\phi$ equations of motion. 2. $J=\delta I[A] / \delta A$, where $I[A]$ is some gaugeinvariant, and Lorentz invariant, functional of $A$. In this case, $D_{\mu} J^{\mu} \equiv 0$. This will lead to higher-derivative additions to the action. There is, however, a third possibility, at least in 3D. In the spin-2 context, this third way is realized by minimal massive gravity (MMG) [3-5], which is a modification of the much-studied topologically massive gravity [6]. What we show here is that there is a spin-1 analog of the construction of Ref. [3], realized as a particular modification of either YM theory (if $\mu=0$ ) or TMYM theory (if $\mu \neq 0$ ).

Consider the current

$$
J^{\mu} \propto \varepsilon^{\mu \nu \rho} \tilde{F}_{\nu} \times \tilde{F}_{\rho} .
$$

This current involves only the gauge field $A$, through its field strength. It is not identically conserved,

$$
D_{\mu} J^{\mu} \propto\left(\varepsilon^{\mu \nu \rho} D_{\mu} \tilde{F}_{\nu}\right) \times \tilde{F}_{\rho} \not \equiv 0,
$$


but using the source-free TMYM equation (4), we find that

$$
D_{\mu} J^{\mu} \propto \mu \tilde{F}^{\mu} \times \tilde{F}_{\mu} \equiv 0 .
$$

In other words, the third possibility is that $J$ is conserved as a consequence of the YM or TMYM equation itself. The obvious difficulty with this idea is that we change the YM or TMYM equation as soon as we include $J$ as a source, but in this case,

$$
D_{\mu} J^{\mu} \propto J^{\mu} \times \tilde{F}_{\mu} \propto \varepsilon^{\mu \nu \rho}\left(\tilde{F}_{\mu} \times \tilde{F}_{\nu}\right) \times \tilde{F}_{\rho} \equiv 0 .
$$

The final identity is a consequence of the Lie algebra Jacobi identity, so the current $J$ of (8) is conserved as a consequence of the YM or TMYM equation even after this equation is modified to include $J$. We have now verified the consistency of the modified equation

$$
\varepsilon^{\mu \nu \rho}\left(D_{\nu} \tilde{F}_{\rho}+\frac{1}{2 m} \tilde{F}_{\nu} \times \tilde{F}_{\rho}\right)+\mu \tilde{F}^{\mu}=0,
$$

where $m$ is a further mass parameter.

It would appear that the new addition to the YM equation breaks parity, even when $\mu=0$, because if the 1 -form $A$ is parity even - as it apparently must be for its field strength 2-form $F$ to have definite parity-then the dual 1-form $\tilde{F}$ is parity odd, implying that its covariant exterior derivative $D \tilde{F}$ is parity odd but also that the 2 -form $\tilde{F} \times \tilde{F}$ is parity even. Nevertheless, Eq. (12) does not break parity when $\mu=0$. To see this one must assign the following parity transformation to the 1 -form $A$ :

$$
\mathcal{P}: A \rightarrow A+m^{-1} \tilde{F} .
$$

The parity transformation of $F$ is then

$$
\mathcal{P}: F \rightarrow F+\frac{1}{m}\left(D \tilde{F}+\frac{1}{2 m} \tilde{F} \times \tilde{F}\right),
$$

so that $\tilde{F}$ is still parity odd, and hence $\tilde{F} \times \tilde{F}$ is still parity even, when one uses the $\mu=0$ equation of motion. The clash with the apparent odd parity of the $D \tilde{F}$ term is resolved by the shift of $A$, which flips the sign of the $\tilde{F} \times \tilde{F}$ term in $D \tilde{F}+(1 / 2 m) \tilde{F} \times \tilde{F}$, so the $\mu=0$ equation of motion preserves parity. We shall see later that the new YM theory can be formulated in a way that makes this feature manifest.

The 3D YM stress tensor is

$$
T_{\mu \nu}=\tilde{F}_{\mu} \cdot \tilde{F}_{\nu}-\frac{1}{2} \eta_{\mu \nu} \tilde{F}_{\rho} \cdot \tilde{F}^{\rho} .
$$

This tensor has the property that $\partial^{\mu} T_{\mu \nu}=0$ as a consequence of either the YM equation or the TMYM equation.
This remains true even if the equation used is the modified version of Eq. (12):

$$
\begin{aligned}
\partial^{\mu} T_{\mu \nu} & =2 \tilde{F}^{\mu} \cdot D_{[\mu} \tilde{F}_{\nu]}=-2 m^{-1} \tilde{F}^{\mu} \cdot \tilde{F}_{\mu} \times \tilde{F}_{\nu} \\
& =-2 m^{-1} \tilde{F}^{\mu} \times \tilde{F}_{\mu} \cdot \tilde{F}_{\nu} \equiv 0 .
\end{aligned}
$$

This suggests that the coupling to $3 \mathrm{D}$ gravity will be straightforward.

The same cannot be said of minimal coupling to lowerspin matter. Consider the equation

$$
\varepsilon^{\mu \nu \rho}\left(D_{\nu} \tilde{F}_{\rho}+\frac{1}{2 m} \tilde{F}_{\nu} \times \tilde{F}_{\rho}\right)+\mu \tilde{F}^{\mu}=\mathcal{J}^{\mu},
$$

where $\mathcal{J}$ is a matter source current. Taking the divergence of this equation and then using it to simplify the result, we deduce that

$$
D_{\mu} \mathcal{J}^{\mu}+m^{-1} \tilde{F}_{\mu} \times \mathcal{J}^{\mu}=0 .
$$

Only when $m^{-1}=0$ can we take $\mathcal{J}$ to be a Noether current $j(\phi)$, so it is not immediately clear whether there is a consistent coupling to lower-spin matter. However, given a covariantly conserved matter current $j(\phi)$, and assuming that $m \neq \mu$, the consistency condition (18) is satisfied by a source current of the form

$$
\begin{aligned}
\mathcal{J}^{\mu}= & j^{\mu}-\frac{1}{(m-\mu)} \varepsilon^{\mu \nu \rho} D_{\nu} j_{\rho}-\frac{1}{m(m-\mu)} \varepsilon^{\mu \nu \rho} \tilde{F}_{\nu} \times j_{\rho} \\
& +\frac{1}{2 m(m-\mu)^{2}} \varepsilon^{\mu \nu \rho} j_{\nu} \times j_{\rho} .
\end{aligned}
$$

Notice that this is quadratic in the covariantly conserved current $j$, in close analogy to the source tensor for MMG, which is quadratic in the matter stress tensor [4]. To verify that Eq. (19) solves Eq. (18), one needs to use the Lie algebra Jacobi identity and Eq. (17), which includes the source.

We shall now consider the particular case of coupling to an adjoint Brout-Englert-Higgs (BEH) field, i.e., a triplet scalar $\phi$ for gauge group $S U(2)$. Assuming that the $\phi$ field equation is

$$
\left[D^{\mu} D_{\mu}+2 V^{\prime}\right] \phi=0,
$$

for potential $V(\phi \cdot \phi)$, the covariantly conserved current is

$$
j_{\mu}=\phi \times D_{\mu} \phi .
$$

In this case,

$$
\begin{aligned}
\mathcal{J}^{\mu} & =\phi \times D^{\mu} \phi-(m-\mu)^{-1} \phi \times\left(\tilde{F}^{\mu} \times \phi\right)+\cdots \\
& =\phi \times\left\{\left[A^{\mu}-(m-\mu)^{-1} \varepsilon^{\mu \nu \rho} \partial_{\nu} A_{\rho}\right] \times \phi\right\}+\cdots,
\end{aligned}
$$


where omitted terms are nonlinear, even when $\phi$ has a nonzero vacuum value. Let us now suppose that

$$
\phi=v+\varphi,
$$

where $v$ is a constant $S U(2)$ triplet and $\varphi$ has zero vacuum value. Then,

$$
\begin{aligned}
v \cdot \mathcal{J}^{\mu} & =0+\cdots, \\
v \times \mathcal{J}^{\mu} & =v^{2}\left[v \times A^{\mu}-(m-\mu)^{-1} \varepsilon^{\mu \nu \rho} \partial_{\nu}\left(v \times A_{\rho}\right)\right]+\cdots,
\end{aligned}
$$

where omitted terms are nonlinear. We see that the vector potential gauging the unbroken $U(1)$ gauge group is unaffected by the $\mathrm{BEH}$ field; it continues to propagate a single spin-1 mode of mass $\mu$. The other two vector potentials each acquire an explicit mass term (with masssquared $v^{2}$ ), so they each propagate a pair of spin-1 modes, but these vector potentials also have a topological mass term, now with mass parameter $\mu+v^{2} /(m-\mu)$; notice that this is nonzero even when $\mu=0$.

In the special case that $\mu=0$, we have, in addition to one massive scalar mode, one massless mode propagated by the $U(1)$ vector potential and four massive modes propagated by the other two vector potentials; each propagates two spin-1 modes of opposite (3D) helicities but with different masses because of the topological mass $\left(v^{2} / m\right)$ induced by the symmetry breaking. In a paritypreserving theory, massive spin-1 modes must appear in parity doublets of opposite helicities, so parity is broken by the coupling to matter for a finite $m$, even though the source-free theory with $\mu=0$ preserves parity. This is a consequence of the fact that $A$ is not parity inert for finite $m$. We shall see later how to modify the construction so as to preserve parity when $\mu=0$.

Although there is no local gauge-invariant action for $A$ alone that yields the source-free equation (12), there is an action involving auxiliary fields, provided that $m \neq \mu$. The Lagrangian density $\mathcal{L}$ is given by

$$
\begin{aligned}
g^{2} \mathcal{L}= & G_{\mu} \cdot \tilde{F}^{\mu}-\frac{1}{2 m}(m-\mu) G_{\mu} \cdot G^{\mu}+\mu \mathcal{L}_{\mathrm{CS}} \\
& +\frac{1}{2 m} \varepsilon^{\mu \nu \rho}\left(G_{\mu} \cdot D_{\nu} G_{\rho}+\frac{1}{3 m} G_{\mu} \cdot G_{\nu} \times G_{\rho}\right) .
\end{aligned}
$$

In the $m \rightarrow \infty$ limit, the auxiliary vector field $G$ [which is also an $S U(2)$ triplet] can be trivially eliminated, and we are then back to the standard TMYM action. More generally, a variation of both $A$ and $G$ induces the following variation of $\mathcal{L}$ :

$$
\begin{aligned}
g^{2} \delta \mathcal{L}= & \frac{(m-\mu)}{m} \delta G_{\mu} \cdot\left(\tilde{F}^{\mu}-G^{\mu}\right)+\left(\delta A_{\mu}+m^{-1} \delta G_{\mu}\right) \\
& \cdot\left[\varepsilon^{\mu \nu \rho}\left(D_{\nu} G_{\rho}+\frac{1}{2 m} G_{\nu} \times G_{\rho}\right)+\mu \tilde{F}^{\mu}\right] .
\end{aligned}
$$

From this result we see that the field equations imply both $G^{\mu}=\tilde{F}^{\mu}$ and a further equation that becomes Eq. (12) upon substitution for $G$.

We also see from Eq. (26) that it is not the $G$ field equation alone that allows us to solve for $G$; that equation also involves $D G$ and a term quadratic in $G$. It is a linear combination of the $A$ and $G$ field equations that allows us to eliminate $G$, but for this reason back substitution in the action is illegitimate. This accords nicely with our earlier conclusion that, as a consequence of its third way construction, the modified YM equation is not the EulerLagrange equation for any local gauge-invariant action constructed from $A$ alone.

Observe that only the $G_{\mu} \cdot G^{\mu}$ term in Eq. (25) involves the 3D Minkowski metric. From this, and the fact that $G=\tilde{F}$ on shell, it follows that the stress tensor is the usual one-i.e., $T_{\mu \nu}$ of Eq. (15) - times a factor of $(m-\mu) / m$. We have already verified that $\partial^{\mu} T_{\mu \nu}=0$ remains true for a finite $m$. We now see that the energy will be positive or negative according to the sign of $m(m-\mu)$, and that positive energy requires

$$
m(m-\mu)>0 .
$$

We may also use Eq. (25) to recover our earlier result (19) for the source current $\mathcal{J}$. We just add to $\mathcal{L}$ the interaction term $-A_{\mu} \cdot j^{\mu}$. This is gauge invariant provided that $D_{\mu} j^{\mu}=0$, but (as will become clear shortly) it breaks parity when $m$ is finite. With this term included, the $A$ equation becomes

$$
\varepsilon^{\mu \nu \rho}\left(D_{\nu} G_{\rho}+\frac{1}{2 m} G_{\nu} \times G_{\rho}\right)+\mu \tilde{F}^{\mu}=j .
$$

Recall now that this equation is needed, in addition to the $G$ equation, to determine $G$, so $G$ will acquire a $j$ dependence. In fact,

$$
G^{\mu}=\tilde{F}^{\mu}+(m-\mu)^{-1} j .
$$

If this is now substituted into the $A$ equation and all $j$-dependent terms are taken to the right-hand side, the result of Eq. (19) is recovered. This construction parallels the construction in Ref. [4] of the source tensor for MMG.

We now show how the parity invariance of the action for $\mu=0$ may be made manifest. First, we introduce the new gauge potential

$$
\bar{A}=A+m^{-1} G,
$$


and we then rewrite the action in terms of $A$ and $\bar{A}$ by using $G=m(\bar{A}-A)$. The result is

$$
\begin{aligned}
I= & \frac{m}{g^{2}} I_{\mathrm{CS}}[\bar{A}]-\frac{(m-\mu)}{g^{2}} I_{\mathrm{CS}}[A] \\
& -\frac{1}{2 g^{2}} m(m-\mu) \int d^{3} x(\bar{A}-A)_{\mu} \cdot(\bar{A}-A)^{\mu} .
\end{aligned}
$$

When $m=\mu$ we may eliminate $A$, trivially, to obtain a Chern-Simons (CS) action for $\bar{A}$, here for gauge group $S U(2)$, but the equations of motion of this CS action are not equivalent to the $m=\mu$ case of Eq. (12), for which special case no action is known.

When $\mu=0$ the action (31) preserves parity if parity is assumed to exchange $A$ with $\bar{A}$. Then, although parity flips the sign of the two CS actions, it also exchanges them, so their difference is parity even provided their coefficients sum to zero, which is the case when $\mu=0$. After elimination of $G$ to recover the new YM field equation of Eq. (12), the parity transformation of $A$ becomes $A \rightarrow \bar{A}=A+m^{-1} \tilde{F}$. It should now be clear how we must proceed if we wish minimal coupling to lower-spin matter to preserve parity when $\mu=0$ : we must choose the gauge potential to be the parity-inert combination

$$
C=\frac{1}{2}(A+\bar{A})=A+\frac{1}{2 m} G,
$$

and we then add the parity-preserving interaction term

$$
\mathcal{L}_{\text {int }}=-C_{\mu} \cdot j^{\mu}, \quad \partial_{\mu} j^{\mu}+C_{\mu} \times j^{\mu}=0 .
$$

Since parity flips the sign of $\mu$ in Eq. (31), we may assume that $\mu \geq 0$. In addition, the field redefinition $A \leftrightarrow \bar{A}$ yields the same action but with $m$ replaced by $\mu-m$, so we may further assume $m \geq \mu / 2$. Thus $m \geq \mu / 2 \geq 0$ may be assumed without loss of generality, but we also need $m>\mu$ for positive energy, in which case (excluding $m=\mu$ ) we have

$$
m>\mu \geq 0,
$$

which excludes $m=0$.

Remarkably, the $\mu=0$ case of the action (31) has appeared previously [7], as a model designed to illustrate a "novel Higgs mechanism" [8]. In this context it arises from a CS theory for gauge group $S U(n) \times S U(n)$ coupled to a bifundamental Higgs field that breaks $S U(n) \times S U(n)$ to the diagonal $S U(n)$ subgroup [here $S U(2)$ ]. This construction yields an additional singlet massive scalar field, so once this is included the model becomes a CS gauge theory minimally coupled to scalar fields, which is renormalizable as a 3D quantum field theory.

If the source-free Lagrangian density (25) is rewritten in terms of $C$ rather than $A$, the result for $\mu=0$ is particularly simple:

$$
g^{2} \mathcal{L}=G_{\mu} \cdot \tilde{H}^{\mu}-\frac{1}{2} G_{\mu} \cdot G^{\mu}+\frac{1}{24 m^{2}} \varepsilon^{\mu \nu \rho} G_{\mu} \cdot G_{\nu} \times G_{\rho},
$$

where

$$
\tilde{H}^{\mu}=\varepsilon^{\mu \nu \rho}\left(\partial_{\mu} C_{\nu}+\frac{1}{2} C_{\mu} \times C_{\nu}\right) .
$$

Parity is manifestly preserved since $C$ is parity even and $G$ is parity odd. This action was also given in Refs. [7,9], where it was observed that the field equation for $G$ can be solved recursively, yielding an infinite series expansion in powers of $1 / \mathrm{m}^{2}$ :

$$
G^{\mu}=\tilde{H}^{\mu}+\frac{1}{8 m^{2}} \varepsilon^{\mu \nu \rho} \tilde{H}_{\nu} \times \tilde{H}_{\rho}+\mathcal{O}\left(m^{-4}\right) .
$$

We may then back substitute to get an action for $C$ alone. We may also substitute for $G$ in the $C$ equation to get (for $\mu=0$ ) an equation for $C$ in the form of an infinite series, but this series is not explicitly defined and will not converge for all values of the dual field strength $\tilde{H}$. In contrast, our simple equation (12) for $A$ is, in addition to being more general, both explicit and defined for all values of $\tilde{H}$; we should note here that a special case $(m=2 \mu)$ has appeared previously in a related context [10].

The Lagrangian density (35) is a convenient starting point for the construction of the Hamiltonian formulation for the $\mu=0$ case. Performing a time-space split we find that

$$
\begin{aligned}
g^{2} \mathcal{L}= & \frac{1}{2} G_{0} \cdot G_{0}+G_{0} \cdot\left(B+\frac{1}{8 m^{2}} \varepsilon_{i j} E^{i} \times E^{j}\right) \\
& +E^{i} \cdot \dot{C}_{i}+C_{0} \cdot\left(\partial_{i} E^{i}+C_{i} \times E^{i}\right)-\frac{1}{2} E_{i} \cdot E_{i},
\end{aligned}
$$

where a sum over $i=1,2$ is implicit, and

$$
E^{i}=\varepsilon^{i j} G_{j}, \quad B=\varepsilon^{i j}\left(\partial_{i} C_{j}+\frac{1}{2} C_{i} \times C_{j}\right) .
$$

The auxiliary field $G_{0}$ may now be trivially eliminated; this yields

$$
g^{2} \mathcal{L}=E^{i} \cdot \dot{C}_{i}+C_{0} \cdot D_{i} E^{i}-H,
$$

where the covariant derivative is now defined with gauge potential $C$ and the Hamiltonian is

$$
H=\frac{1}{2} E_{i} \cdot E_{i}+\frac{1}{2}\left|B+\frac{1}{8 m^{2}} \varepsilon^{i j} E_{i} \times E_{j}\right|^{2} .
$$


Here, $|\ldots|$ is the $S U(2)$-triplet norm. We see that the canonical variables $\left\{C_{i}, E^{i}\right\}$ are subject to the Gauss-law constraint $D_{i} E^{i}=0$, as in the standard Hamiltonian formulation of 3D YM theory. The only difference is in the Hamiltonian, which includes additional terms. Notice, however, that these are such that the Hamiltonian remains manifestly positive.

For the generic case of nonzero $\mu$, it is simpler to perform a time-space split in the action (31). Provided that $m(m-\mu) \neq 0$ we can then trivially eliminate $\left(A_{0}-\bar{A}_{0}\right)$ to get

$$
\begin{aligned}
\mathcal{L}= & \frac{(m-\mu)}{2 g^{2}} \varepsilon^{i j} A_{i} \cdot \dot{A}_{j}-\frac{m}{2 g^{2}} \varepsilon^{i j} \bar{A}_{i} \cdot \dot{\bar{A}}_{j} \\
& +\frac{1}{g^{2}} C_{0} \cdot[m \bar{B}+(m-\mu) B]-H,
\end{aligned}
$$

where

$$
\begin{aligned}
H= & \frac{1}{2 g^{2}}\left[m(m-\mu)\left(A_{i}-\bar{A}_{i}\right) \cdot\left(A_{i}-\bar{A}_{i}\right)\right. \\
& \left.+\frac{1}{m(m-\mu)}|m \bar{B}-(m-\mu) B|^{2}\right] .
\end{aligned}
$$

Here, $B$ is defined as in Eq. (39), and $\bar{B}$ is the same but with $\bar{A}$ instead of $A$. The field $C_{0}$ is again the time component of the parity-even gauge potential $C$, and it is again a Lagrange multiplier for an $S U(2)$ triplet of first-class constraints, which generate $S U(2)$ gauge transformations of the canonical variables. Notice that the Hamiltonian is positive only if $m(m-\mu)>0$, as expected from our earlier discussion of the stress tensor.

We conclude with a comment on the relation of our construction to $M$-theory. We defer to Ref. [9] for a review of the relevance to multi-M2-brane dynamics of the action (31) for $\mu=0$. Its relevance for $\mu \neq 0$ follows from the work of Ref. [11], where the sum of the CS levels was identified with the Romans mass of massive IIA supergravity [12]. In our construction, this sum is proportional to the mass $\mu$ of our modified TMYM equation. This is in accord with the fact that consistency of the topologically massive super-D2-brane in a supergravity background implies the field equations of massive IIA supergravity [13].

We are grateful to Sunil Mukhi for his comments on a draft of this paper, for bringing Ref. [10] to our attention, and for sending us his unpublished notes, which contain some of the results presented here. We also thank Stanley Deser and Bengt Nilsson for their helpful correspondence. A. S. A. and P. K. T. acknowledge support from the United Kingdom Science and Technology Facilities Council (Grant No. ST/L000385/1). A. S. A. also acknowledges support from Clare Hall College, Cambridge, and from the Cambridge Trust. A. S is supported in part by the Belgian Federal Science Policy Office through the Interuniversity Attraction Pole P7/37, and in part by the FWO-Vlaanderen through Project No. G020714N and by the Vrije Universiteit Brussel through the Strategic Research Program "HighEnergy Physics".

*A.S.Arvanitakis@damtp.cam.ac.uk

†Alexandre.Sevrin@vub.ac.be

p.k.townsend@damtp.cam.ac.uk

[1] J. F. Schonfeld, A mass term for three-dimensional gauge fields, Nucl. Phys. B185, 157 (1981).

[2] S. Deser, R. Jackiw, and S. Templeton, Three-Dimensional Massive Gauge Theories, Phys. Rev. Lett. 48, 975 (1982).

[3] E. Bergshoeff, O. Hohm, W. Merbis, A. J. Routh, and P. K. Townsend, Minimal massive 3D gravity, Classical Quantum Gravity 31, 145008 (2014).

[4] A. S. Arvanitakis, A. J. Routh, and P. K. Townsend, Matter coupling in 3D minimal massive gravity, Classical Quantum Gravity 31, 235012 (2014).

[5] A. S. Arvanitakis and P. K. Townsend, Minimal massive 3D gravity unitarity redux, Classical Quantum Gravity 32, 085003 (2015).

[6] S. Deser, R. Jackiw, and S. Templeton, Topologically massive gauge theories, Ann. Phys. (N.Y.) 140, 372 (1982); 185, 406(E) (1988); 281, 409(E) (2000).

[7] S. Mukhi, Unravelling the novel Higgs mechanism in $(2+1)$ d Chern-Simons theories, J. High Energy Phys. 12 (2011) 083.

[8] S. Mukhi and C. Papageorgakis, M2 to D2, J. High Energy Phys. 05 (2008) 085.

[9] J. Bagger, N. Lambert, S. Mukhi, and C. Papageorgakis, Multiple membranes in M-theory, Phys. Rep. 527, 1 (2013).

[10] B. E. W. Nilsson, Critical solutions of topologically gauged $N=8$ CFTs in three dimensions, J. High Energy Phys. 04 (2014) 107.

[11] D. Gaiotto and A. Tomasiello, The gauge dual of Romans mass, J. High Energy Phys. 01 (2010) 015.

[12] L. J. Romans, Massive $N=2 a$ supergravity in tendimensions, Phys. Lett. 169B, 374 (1986).

[13] E. Bergshoeff, P. M. Cowdall, and P. K. Townsend, Massive IIA supergravity from the topologically massive D2-brane, Phys. Lett. B 410, 13 (1997). 